\title{
Vitamins at the heart of heart disease?
}

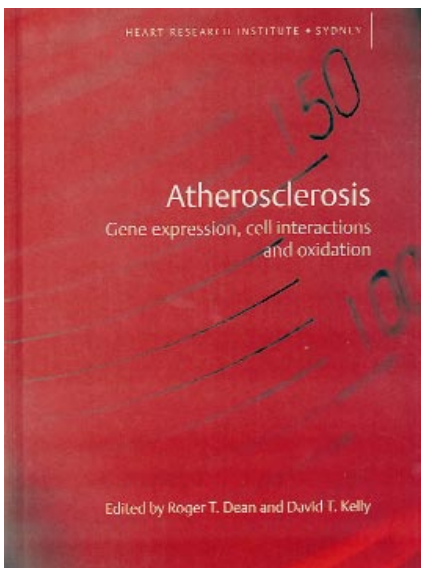

\author{
Atherosclerosis: Gene Expression, Cell Interactions and Oxidation \\ edited by Roger T. Dean and David T. Kelly \\ Oxford University Press · September 2000 \\ Hardback $£ 75 / \$ 130$
}

Kelly L. Krass and Aldons J. Lusis

A therosclerosis is a chronic inflammatory disease of the large arteries that is the cause of heart disease and stroke. Although effective drugs for the treatment of atherosclerosis have been developed, including the cholesterol-lowering family of statins, it remains the leading cause of death in westernized countries. In fact, atherosclerosis is now supplanting infectious disease as the major cause of death worldwide due to the continued westernization of parts of the world. It is a disease of exceptional aetiological complexity, as more than a dozen important risk factors, both genetic and environmental, have been identified in epidemiological studies. It involves a host of cell types, including endothelial cells, monocytes, macrophages, smooth-muscle cells, lymphocytes and platelets, that participate in the initiation, growth and rupture of an atherosclerotic plaque.

Atherosclerosis is a collection of twenty essays focusing on the role of oxidation and inflammation in the disease. The oxidative hypothesis proposes that oxidative processes, particularly those involving lipid oxidation, trigger inflammatory events and promote the formation of the cholesterolengorged macrophages that are a hallmark of the disease.

Over the past decade, the belief that eating a diet low in cholesterol and saturated fats can largely prevent atherosclerosis has become quite popular. Studies have repeatedly shown that high intake of saturated fat is positively related to high mortality from coronary heart disease (CHD). How, then, do we explain the French paradox? France, notorious for its rich foods high in both cholesterol and saturated fatty acids, has an extremely low morbidity due to CHD. Multiple groups have explained this paradox by noting the increased anti-oxidants present in both wine and vegetables consumed regularly by the French. As suggested in Atherosclerosis, perhaps our ability to prevent and reverse oxidative events is more important than the sheer number of lipidrich particles present.
Assuming that antioxidants are the key to healthy arteries, shouldn't we all rush to our nearest vitamin retailer, and purchase large quantities of vitamin $\mathrm{E}$ and $\beta$-carotene? Despite supporting evidence from a variety of animal studies, the hypothesis remains controversial, as some large trials with antioxidants have yielded inconclusive evidence in humans. It is possible that the inability of natural and synthetic antioxidants to markedly slow or stop the progression of human atherosclerotic lesions is because oxidation is merely one of many causative factors, or simply that the administered antioxidants cannot provide the needed protection at the relevant sites, such as the vessel wall.

Atherosclerosis takes a direct and indepth approach to addressing some important questions in atherogenesis. It provides a critical and up-to-date overview of data relating to oxidation, along with useful background information not found in research papers. The book includes an excellent chapter on the results of clinical trials involving antioxidants. It also contains some chapters addressing related aspects of atherogenesis that provide the reader with an understanding of the pathology of the disease. For example, a complete chapter is dedicated to each of the four main cell types involved in the atherogenic process.

Although the book succeeds in exposing the cutting edge of a number of research issues in atherogenesis, its scope is limited because some important areas are omitted. In particular, genetic approaches in humans and animal models are only briefly mentioned. This seems curious, because population association studies with such genetic factors as serum paraoxonase provide some of the more compelling evidence of a link between oxidation and atherosclerosis in humans. There is also little discussion of the hundreds of transgenic mouse studies that have provided strong mechanistic information in vivo, including aspects relevant to oxidation and inflammation. The focus on biochemistry and cellular interactions seems to reflect the research emphasis of The Heart Research Institute, Sydney, where the book originates.

A weakness of the book is the near absence of figures associated with complex passages of text. This lack of pictures is perhaps most notable in the introductory chapter on the pathogenesis of the disease, making Atherosclerosis less suitable for casual readers. With a heavy emphasis on basic research, this book will be of greatest value to investigators involved in cardiovascular research.

After reading the book, we had a much better understanding of the current status of the oxidation hypothesis. Clearly there is a large quantity of supporting data, primarily from studies of tissue culture cells and animal models. However, the French paradox is still a paradox, and purchasing shares in a vitamin distribution company might still be a little premature.

Kelly L. Krass and Aldons J. Lusis are at the Department of Microbiology, Immunology and Molecular Genetics, UCLA, Los Angeles, California 90095, USA e-mail:jlusis@mednet.ucla.edu

\section{New in hardback}

Recent Advances in the Biochemistry of Plant Lipids edited by J. L. Harwood and P. J. Quinn Portland Press, $£ 75$

Neuronal Signal Transduction and Alzheimer's Disease edited by C. O'Neil and B. Anderton Portland Press, $£ 65 / \$ 107.50$

Cell Adhesion and Migration in Skin Disease

edited by J. Barker and J. McGrath

Harwood Academic Publishers, $\mathfrak{E} 67 / \$ 102$

\section{New in paperback}

RNA Editing

edited by Brenda L. Bass

Oxford University Press, £32.50/\$55 